\title{
Die Berücksichtigung des Verschleppungsfehlers bei der Natrium-, Kalium- und Chloridbestimmung mit dem Analysenautomat C 4
}

\author{
Von R. Haeckel und A. J. Porth \\ Institut für Klinische Chemie (Gescbäftsfübrender Direktor: Prof. Dr. Dr. J. Büttner) \\ Medizinische Hocbscbule Hannover
}

(Eingegangen am 3. September 1971)

Der Gesamtverschleppungsfehler bei der simultanen Natrium-, Kalium- und Chloridbestimmung mit dem Analysenautomaten $C 4$ wurde untersucht. Zur Ermittlung des Konzentrationsbereiches, in dem Verschleppungsfehler vernachlässigt werden können, wurde eine mathematische Beziehung zwischen dem Verschleppungskoeffizienten und der methodischen Streuung abgeleitet. Dabei wurde postuliert, daß der Verschleppungsfehler kleiner als der zweifache Bereich der methodischen Streuung sein soll. Mit Hilfe einer graphischen Darstellung läßt sich rasch entscheiden, ob sich Meß- und verschleppungssicherer Bereich decken.

Treatment of the error due to overspill in the determination of sodium, potassium and chloride with the automatic analyser $C 4$ The total error due to overspill in the simultaneous determination of sodium, potassium and chloride with the automatic analyser $C-4$ was investigated. In order to determine the concentration range in which the overspill error can be ignored, a mathematical relationship was derived between the coefficient of overspill and the methodical scatter. It was thus postulated that the overspill error should be smaller than twice the range of methodical scatter. With the aid of a graph, it can be quickly decided whether there is overlap between the ranges of measurement and overspill.

Bei mechanisierten Analysenverfahren kann es zu Verschleppungseffekten kommen, wenn gelöste Reaktionspartner, die sich von verschiedenen Proben ableiten, einen gemeinsamen Weg nehmen. Am Analysenautomat C 4 sind zwei Arten der Verschleppung möglich: eine zyklische, die bei den Elektrolytbestimmungen nicht nachweisbar ist (1) und eine Verschleppung von Probezu-Probe. Die letztere kann bei der Probenaufnahme und bei der Überführung der Reaktionslösungen in das Photometer auftreten. Im folgenden soll untersucht werden, inwieweit die Analysenergebnisse bei der Natrium-, Kalium- und Chloridbestimmung durch die Gesamtverschleppung verfälscht werden.

\section{Methodik}

Die Methodik sowie die Zuverlässigkeit der Analysen wurde in der vorangehenden Mitteilung (1) beschrieben.

Um die Verschleppung zu bestimmen, wurden in der Serie nacheinander 5 Proben mit niedrigen Konzentrationen (Natrium

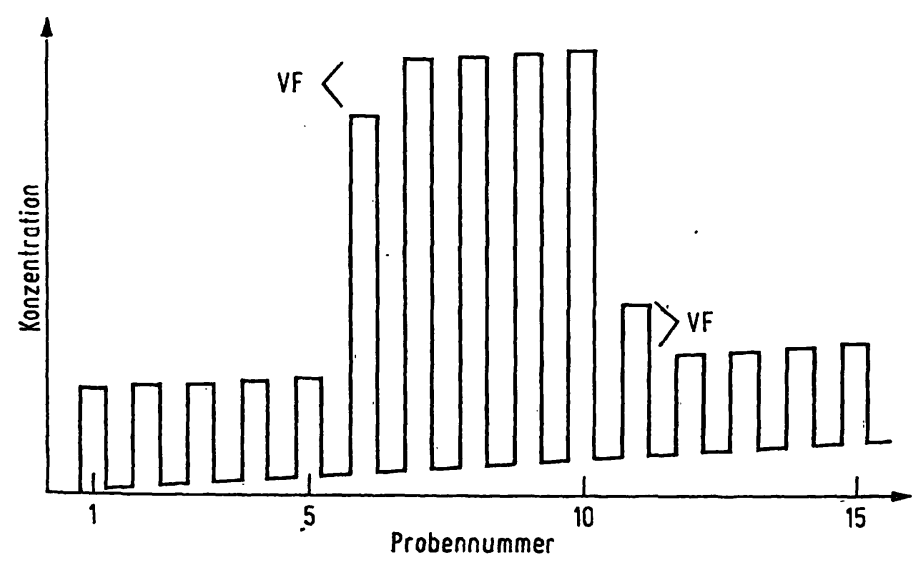

Abb. 1

Probenfolge für die experimentelle Ermittlung des Verschleppungs-
$16 \mathrm{mMol} / \mathrm{l}$, Kalium $0,8 \mathrm{mMol} / \mathrm{l}$, Chlorid $16,8 \mathrm{mMol} / \mathrm{l}), 5 \mathrm{mit}$ hohen Konzentrationen (Natrium $160 \mathrm{mMol} / \mathrm{l}$, Kalium $8 \mathrm{mMol} / \mathrm{l}$, Chlorid $168 \mathrm{mMol} / \mathrm{l}$ ) und zuletzt wieder $5 \mathrm{mit}$ niedrigen Konzentrationen analysiert (Abb. 1). Aus den dabci erhaltenen Ergebnissen $\left(c_{1}\right.$ bis $c_{15}$ ) läßt sich der Verschleppungskoeffizient nach HJELM (2) folgendermaßen berechnen:

$$
\begin{aligned}
& q_{1}=\frac{\bar{x}\left(c_{7} \text { bis } c_{20}\right)-c_{6}}{c_{6}-\bar{x}_{\left(c_{2} b i s c_{6}\right)}} \begin{array}{c}
\text { von der niedrigen zur hohen } \\
\text { Konzentration) }
\end{array} \\
& q_{2}=\frac{\left.c_{11}-\bar{x}_{\left(c_{12}\right.} \text { bis } c_{18}\right)}{\bar{x}_{\left(c_{7} \text { bis } c_{10}\right)}-c_{11}} \begin{array}{c}
\text { von der hohen zur niedrigen } \\
\text { Konzentration) }
\end{array}
\end{aligned}
$$

\section{Ergebnisse}

Albuminhaltige Lösungen verschleppen etwa 1,5 bis 5 fach stärker als eiweißfreie (Tabelle 1). Bei Kalium und Natrium ist die Verschleppung von niedrigen zu hohen geringer als die von hohen zu niedrigen Konzentrationen. Bei der Chloridmethode können sich aufeinander folgende Proben beträchtlich beeinflussen. Daher mußte untersucht werden, ob Konzentrationsunterschiede, die im Extremfall im Serum vorkommen, einen Fehler verursachen können, der im Vergleich zur methodischen Streuung $\mathrm{zu}$ hoch ist.

Hierfür ist zu fordern: Der durch Verschleppungseffekte bedingte Fehler soll kleiner sein als der zweifache Bereich

Tab. 1

Der Einfluß von Albumin auf den Verschleppungskoeffizienten nach

\begin{tabular}{|c|c|c|c|c|}
\hline $\begin{array}{c}\text { Albumin- } \\
\text { konzentration }\end{array}$ & $Q_{1}$ & $Q_{2}$ & $Q_{1}$ & $Q_{2}$ \\
\hline $\begin{array}{l}\text { Chlorid } \\
\text { Kalium } \\
\text { Natrium }\end{array}$ & $\begin{array}{c}\left.2,9 \pm 1,1^{1}\right) \\
0,4 \pm 0,6 \\
\left.?^{2}\right)\end{array}$ & $\begin{array}{l}2,4 \pm 0,7 \\
1,0 \pm 0,5 \\
0,3 \pm 0,3\end{array}$ & $\begin{array}{l}4,8 \pm 1,0 \\
1,3 \pm 1,0 \\
0,9 \pm 0,9\end{array}$ & $\begin{array}{l}3,9 \pm 0,5 \\
1,5 \pm 0,5 \\
1,7 \pm 1,1\end{array}$ \\
\hline
\end{tabular}
HJELM bei den Elektrolytbestimnuungen an Analysenautornat C 4

2) Mittelwerte mit Standardabweichung aus 10 Analysen an verschiedenen Tagen. Verwendetc Konzentrationen: Siehe unter Methodik. ) Nicht sicher nachweisbar. 
der Streuung in der Serie (Standardabweichung). Das bedeutet: Folgt auf eine Probe mit einer hohen Konzentration $\left(c_{1}\right)$ eine zweite mit einer niedrigen Konzentration $\left(c_{2}\right)$, dann soll deren Analysenergebnis den Wert $c_{2}+2 s$ nicht übersteigen. Dabei bedeutet s die Standardabweichung des Mittelwertes aus einer Serienanalyse von $\mathrm{c}_{2}$.

Der Verschleppungskoeffizient nach HJELM (2) kann dann folgendermaßen berechnet werden:

$$
\mathrm{q}_{2}=\frac{2 \mathrm{~s}}{\mathrm{c}_{1}-\left(\mathrm{c}_{2}+2 \mathrm{~s}\right)} \text {. }
$$

Hierbei soll - wie von HJELM (2) ebenfalls implizit vorausgesetzt - gelten: $c_{1}>c_{2}+2 s$. Ferner wird für die folgenden Betrachtungen als gegeben angenommen, $\mathrm{da} S \mathrm{~s}$ in der Praxis größer als Null ist.

Ersetzt man $\mathrm{q}_{2}$ durch den prozentualen Verschleppungskoeffizienten

$$
\mathrm{Q}_{2}=\mathrm{q}_{2} \cdot 100,
$$

so erhält man durch Umformung von (III)

$$
\mathrm{F}_{2}=\frac{\mathrm{c}_{1}}{\mathrm{c}_{2}}=\frac{2 \mathrm{~s}}{\mathrm{c}_{2}} \cdot \frac{\left(100+\mathrm{Q}_{2}\right)}{\mathrm{Q}_{2}}+1 \text {. }
$$

Mit Hilfe dieser Formel (V) kann für jede Methode festgestellt werden, um welchen Faktor die Konzentration einer vorangehenden Probe größer sein darf als die der folgenden Probe, ohne daß die Verschleppung berücksichtigt werden muß.

Setzt man z. B. in die Formel (V) die Werte $c_{2}=88 \pm 1,2$ (1) und $\mathrm{Q}_{2}=3,9$ (Tab. 1) ein, so wird $\mathrm{F}_{2}=1,7$. Daher kann bei Chloridwerten $z$ wischen 88 und $150(=1,7 \times 88)$ $\mathrm{mMol} / \mathrm{l}$ die Verschleppung von hohen zu niedrigen Konzentrationen vernachlässigt werden. Es kann aber auch jeder beliebige Mittelwert mit dem dazugehörigen $s$-Wert innerhalb des gültigen Meßbereiches verwendet werden, da $\mathrm{Q}_{2}$ konzentrationsunabhängig ist (Abb. 2). Zur Ermittlung der hohen Grenzkonzentration $\mathrm{F}_{2} \cdot \mathrm{c}_{2}$ verwendet man die Formel

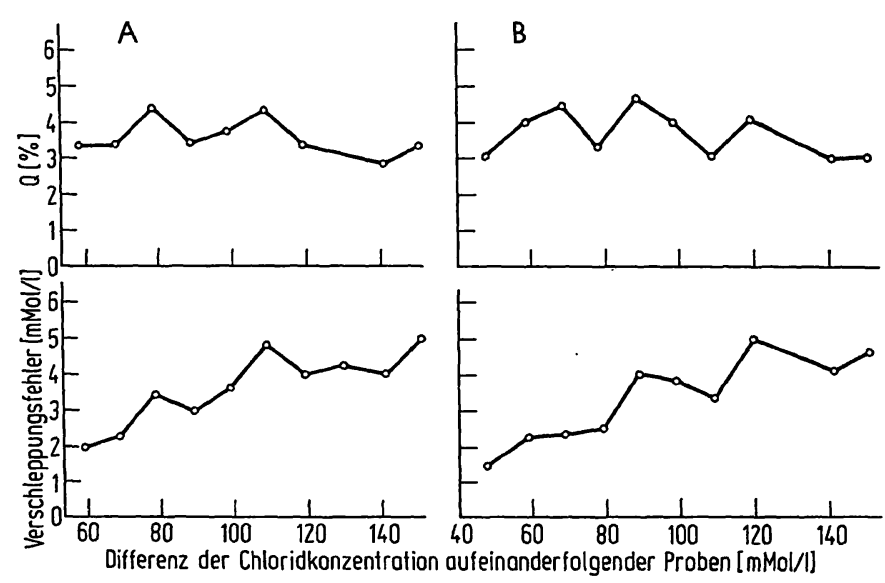

Abb. 2

Die Abhängigkeit des Verschleppungskoeffizienten $Q(\%)$ und des absoluten Verschleppungsfehlers (mMol/l). von der Konzentrationsdifferenz aufeinanderfolgender Proben, bei A von der niedrigen zur hohen und bei $B$ von der hohen zur niedrigen Konzentration. Eine Serie albuminhaltiger Chloridlösungen ( $160 \mathrm{mMol} / \mathrm{l})$ wurde nach je 4 Proben mit niedrigeren Konzentrationen ( 10 bis $100 \mathrm{mMol} / \mathrm{l}$ ) in regelmäßigen Abständen unterbrochen. Der Verschleppungskoeffizient wurde nach Formel (I) und (II) berechnet. Probenaufnahmevolumen: $100 \mu \mathrm{l}$

$$
c_{1,1}=\frac{2 s\left(100+Q_{2}\right)}{Q_{2}}+c_{2} .
$$

In Analogie zu diesen Berechnungen kann auch der zulässige Bereich für die Verschleppung von einer niedrigen $\left(c_{2}\right) z u$ einer hohen $\left(c_{1}\right)$ Konzentration errechnet werden:

$$
\begin{gathered}
F_{1}=\frac{c_{2}}{c_{1}}=1-\frac{2 s}{c_{1}} \cdot \frac{\left(100+Q_{1}\right)}{Q_{1}} . \\
c_{2,1}=c_{1}-\frac{2 s\left(100+Q_{1}\right)}{Q_{1}} .
\end{gathered}
$$

Setzt man die experimentell ermittelten Daten $c_{1}=$ $115 \pm 1,2$ (1) und $\mathrm{Q}_{1}=4,8$ (Tab. 1) in Formel (VIII) ein, so erhält man 63. Daraus folgt, daß zwischen 63 und $115 \mathrm{mMol} / \mathrm{l}$ Chlorid die Verschleppung von niedrigen $\mathrm{zu}$ hohen Konzentrationen vernachlässigt werden kann.

In den Abbildungen 3a bis $3 \mathrm{~b}$ ist der zulässige Bereich für verschiedene Konzentrationen mit den Formeln (VI) und (VIII) berechnet und graphisch dargestellt worden.

Geht man von einem Punkt der Winkelhalbierenden (c) in Pfeilrichtung nach oben (Abb. 3) bis zur Grenzlinie $\left(c_{1}, 1\right)$, so erhält man die maximale Konzentration $\left(c_{1}\right)$, die $c_{2}$ vorangehen darf, damit der absolute Verschleppungsfehler kleiner als $2 \cdot s_{\mathbf{c}_{2}}$ wird. Umgekehrt gibt der Pfeil nach unten die minimale Konzentration $\left(c_{2}\right)$ an, die $c_{1}$ vorangehen darf, damit der Verschleppungsfehler kleiner als $2 \cdot s_{c_{1}}$ ist. Verbindet man diese nach Formel (VI) und (VIII) ermittelten Grenzkonzentrationen, so erhält man einen Grenzbereich (gestrichelte Linie in Abb. 3), in dem Verschleppungseffekte vernachlässigt werden können.

Der für Humanseren gültige Normalbereich und möglichst auch der Extrembereich sollten innerhalb dieses verschleppungssicheren Bereiches liegen.

Während dies für die Natriumbestimmung zutrifft, liegen die Extrembereiche bei Chlorid und Kalium teilweise außerhalb des verschleppungssicheren Bereiches. Folgt z. B. auf eine Probe mit hoher Kaliumkonzentration (über $5 \mathrm{mMol} / \mathrm{l}$ ) eine $z$ weite mit niedrigerer Konzentration (unter $5 \mathrm{mMol} / \mathrm{l}$ ), so kann es zu einem Verschleppungsfehler kommen, der größer ist als der $2 \mathrm{~s}$-Bereich der Streuung in der Serie; er kann maximal $6 \%$ betragen.

\section{Diskussion}

Verschleppungseffekte kann man auf drei Arten korrigieren:

1. Werden Doppelanalysen durchgeführt, so verwirft man den ersten Wert als möglicherweise verfälscht.

2. Rechnerische Korrektur: Nach einem Vorschlag von HJELM (2) werden zu Beginn einer Serie mit 4 Standards der tägliche Verschleppungskoeffizient ermittelt und dann mit Hilfe der Formel

$A_{n}^{*}=A_{n}-q\left(A_{n}-A_{n-1}\right)$ alle Einzelwerte korrigiert.

3. Wiederholung der verfälschten Analysen. 


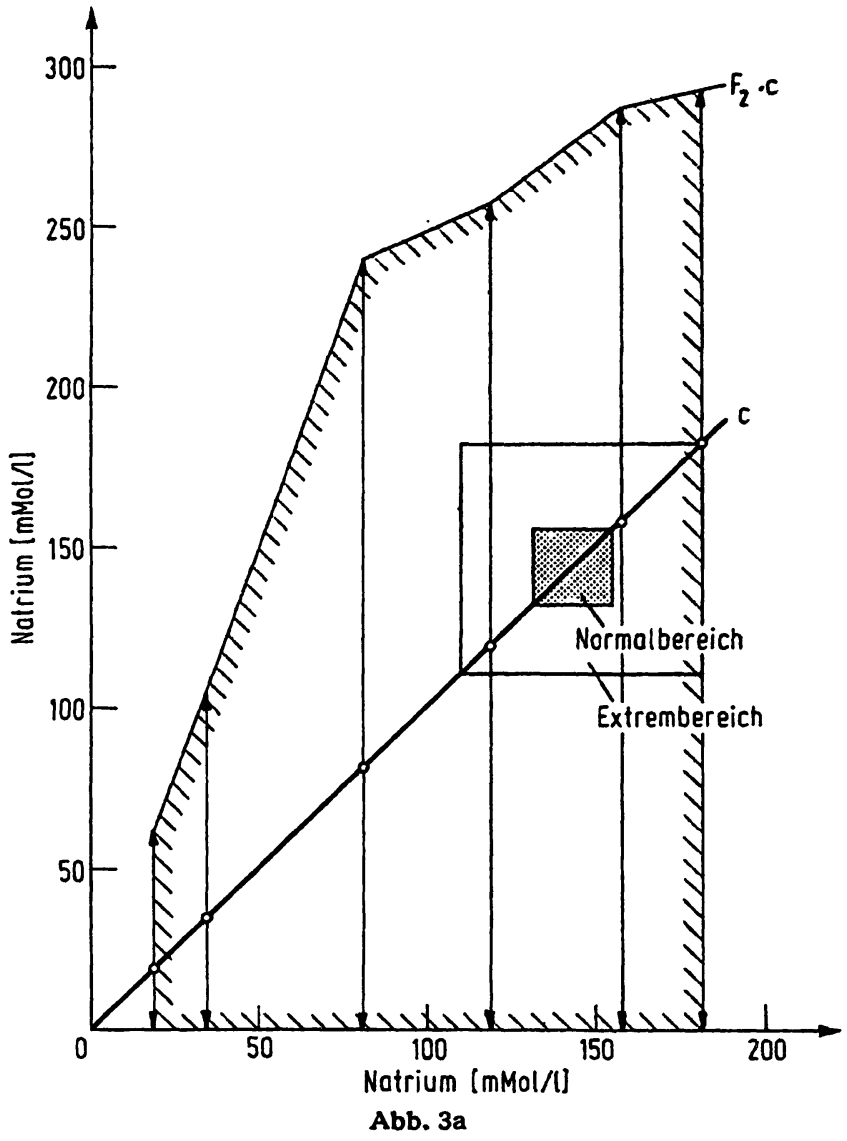

Für ein klinisch-chemisches Routinelaboratorium ist die erste Möglichkeit zu aufwendig, und scheitert in der Praxis oft an der geringen Probemenge. Die zweite ist u. E. an einen Computer gebunden und nur durchführbar, wenn der vorangegangene hohe Wert im Meßbereich liegt. Außerdem wird sie dann kompliziert, wenn $q_{1}$ und $\mathrm{q}_{2}$ verschieden groß sind. Das dritte Verfahren erfordert in der Praxis, insbesondere wenn keine elektronische Datenverarbeitung zur Verfügung steht, den geringsten Aufwand. Allerdings müssen dazu Kriterien bekannt sein, wann eine Analyse wiederholt werden muß. Diese können nach der oben beschriebenen Methode einmal experimentell in einer mehrwöchigen Versuchsserie ermittelt werden.

Sind die mittleren Verschleppungskoeffizienten aus mehreren Experimenten, sowie die Streuung in der Serie von einigen Konzentrationen im unteren und oberen Meßbereich bekannt, kann mit Hilfe von Formel (VI) und (VIII) der Konzentrationsbereich ermittelt werden, in dem der Verschleppungseffekt vernachlässigt werden kann. Dabei wird postuliert, daß der Verschleppungsfehler kleiner als der zweifache Bereich der methodischen Streuung sein soll.

Die Methoden sollten in der klinischen Chemie so ausgelegt werden, daß der Meß-bzw. Extrembereich innerhalb des verschleppungssicheren Bereiches liegt. Soweit uns bekannt, wurde bisher kein Verfahren mitgeteilt,
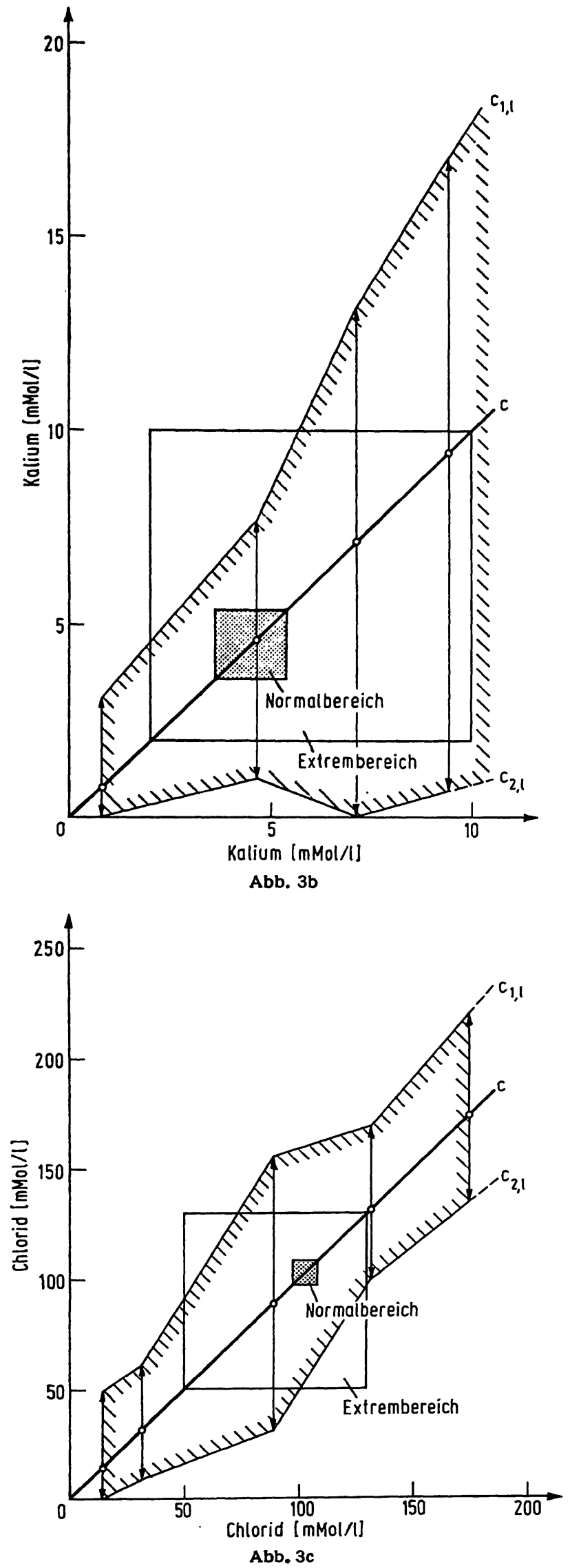

Abb. 3a, 3b, 3c

Der verschleppungssichere Bereich (gestrichelte Linie) bei der Natrium(a), Kalium-(b) und Chloridbestimmung (c)

Die Grenzkonzentrationen für die Verschleppung von niedrigen zu hohen $\left(c_{1}, 1\right)$ und von hohen zu niedrigen Konzentrationen $\left(c_{2,1}\right)$ wurden nach Formel (6) und (8) zu den durch Kreise markierten Konzentrationen berechnet. Die Verschleppungskoeffizienten wurden der Tabelle 1 und die entsprechenden Werte für die Streuung in der serie aus (1) entnommen 
mit dem dies geprüft werden kann. Die oben beschriebene Methode ermöglicht mit einem Blick festzustellen, ob Verschleppungsfehler berücksichtigt werden müssen. Ist dies der Fall, so ersieht man aus der graphischen Darstellung des verschleppungssicheren Bereiches (Abb. 3) leicht, wann eine Analyse wiederholt werden muß. Eine Bestimmung wird grundsätzlich nochmals durchgeführt, wenn die Konzentration der vorangehenden Probe außerhalb des gültigen Meßbereiches liegt.

Die unregelmäßigen Abweichungen der Begrenzungslinien bei der graphischen Darstellung des verschleppungssicheren Bereiches sind methodisch bedingt. In folgenden Versuchsreihen werden weitere Daten gesammelt, mit deren Hilfe eine mathematische Ableitung dieser Grenzen angestrebt wird. Außerdem sollen die graphischen Darstellungen zu Nomogrammen erweitert werden, aus denen man die korrigierten Werte (außerhalb des verschleppungssicheren Bereiches) direkt ablesen kann.

Das von uns für Routinelaboratorien vorgeschlageñe Konzept besteht also darin, für jede Methode (bei der es zu Verschleppungsfehlern kommen kann) einen verschleppungssicheren Bereich experimentell zu ermitteln. Bei Konzentrationen außerhalb dieses Bereiches, die in der Praxis selten vorkommen sollten, muß die Analyse wiederholt oder das Ergebnis korrigiert werden.

Fräulein GesIne KNIrTER danken wir für die technische Durchführung der Experimente.

\section{Literatur}

1. HAECKEL, R., diese Z. 10, 86 (1972). - 2. HJeLM, M., Z. analyt. Chem. 243, 781 (1968).

Priv. Doz. Dr. R. Haeckel

3000 Hannover

Roderbruchstr. 101

Dipl.-Math. Dr. A. J. Porth

Arbeitsgruppe "Automation und EDV im Laboratorium“ 3000 Hannover

Podbielskistr. 380 